\title{
Balmer line shifts in quasars
}

\author{
J. W. Sulentic • P. Marziani • A. Del Olmo • \\ S. Zamfir
}

\begin{abstract}
We offer a broad review of Balmer line phenomenology in type 1 active galactic nuclei, briefly summarising luminosity and radio loudness effects, and discussing interpretation in terms of nebular physics along the $4 \mathrm{D}$ eigenvector 1 sequence of quasars. We stress that relatively rare, peculiar Balmer line profiles (i.e., with large shifts with respect to the rest frame or double and multiple peaked) that start attracted attentions since the 1970s are still passable of multiple dynamical interpretation. More mainstream objects are still not fully understood as well, since competing dynamical models and geometries are possible. Further progress may come from inter-line comparison across the 4D Eigenvector 1 sequence.
\end{abstract}

Keywords galaxies: active; quasars: emission lines; quasars: general

\section{Introduction: Quasars and line shifts}

Internal line shifts in quasar spectra have played an ever-increasing role in our understanding of quasar structure and dynamics. Apart from early attempts to explain quasar cosmological redshifts as intrinsic to the quasar (see e.g., the historical account in Chapter 2 of D'Onofrio et al. 2012) that lead to serious inconsistency (Greenstein and Schmidt 1964), the realization that all lines in a quasar do not yield the same

J. W. Sulentic

Instituto de Astrofísica de Andalucía (CSIC), Granada, Spain

P. Marziani

INAF, Osservatorio Astronomico di Padova, Padova, Italia

A. Del Olmo

Instituto de Astrofísica de Andalucía (CSIC), Granada, Spain

S. Zamfir

University of Wisconsin, Stevens Point, WI, USA redshift was slow to come. Until the early 1980s and the pioneering paper by Martin Gaskell (Gaskell 1982) redshift difference between emission lines had not been systematically studied in quasars, even if some cases of broad line shifts $\left(\sim 1000 \mathrm{~km} \mathrm{~s}^{-1}\right.$ with respect to narrow lines) had been noted in Seyfert galaxies (Osterbrock 1979). Gaskell's results on the difference between low- and high-ionization lines (LILs and HILs, represented by MgII $\lambda 2800$ and CIV $\lambda 1549$ respectively) have remained valid since then, and have yielded the observational basis for a model that is still today providing a basic interpretative sketch for the structure of the broad line emitting region (Collin-Souffrin et al. 1988). In that model, HIL blue shifts are associated with radial motion in a flattened structure (the accretion disk) emitting mainly LILs. Later developments (Tytler and Fan 1992, Marziani et al. 1996, Corbin and Boroson $1996)$ confirmed the earlier results. Sulentic et al. (1995) suggested that results were not the same for all quasars, and that a first distinction between radio-loud and radio-quiet quasars was necessary: blueshifts were mostly present in radio quiet (RQ) quasars, but definitely rarer or of lower amplitudes in radio loud (RL). This finding (confirmed in Marziani et al. 1996 and Richards et al. 2011) has been further contextualised within the Pop. A and B concept in the 4D Eigenvector 1 formalism (e.g. Sulentic et al. 2000, 2007, 2011). In the following we will focus attention on the $\mathrm{H} \beta$ line, as representative of LILs, and its contextualization within $4 \mathrm{DE} 1(\$ 2)$. We report the observational properties in a systematic way $(\$ 3)$ that includes a robust empirical interpretation based on nebular physics $(\$ 3.1$, and effects of luminosity and radio loudness $(\$ 3.2)$. We then discuss the physical processes that may be relevant in line profile broadening ( $\$ 4$. We conclude with remarks on unknown aspects and desiderata for further observations $(\$ 5$ and $\S 6$. 
2 (4D)Eigenvector 1 quasar contextualization at low- $z$

The (4D) Eigenvector 1 quasar contextualization scheme at low- $z$ offers a powerful tool for interpretation of the $\mathrm{H} \beta$ emission line (and of LILs in general) profiles. Eigenvector 1 was originally defined from a Principal Component Analysis (PCA) of 87 PG quasars involving an anticorrelation between optical FeII intensity, half-maximum profile width of $\mathrm{H} \beta$ and peak intensity of [OIII] $\lambda 5007$ (Boroson and Green 1992). E1 was afterwards expanded to 4DE1 with the addition of X-ray photon index and CIV $\lambda 1549$ profile shift measures ( $\mathrm{Su}-$ lentic et al. 2000, 2008, 2011). 4DE1 allows one to define a quasar main sequence in 4DE1 space (Sulentic et al. 2000, Marziani et al. 2001).

The principle parameters of the 4DE1 space are:

- Full width at half maximum of broad $\mathrm{H} \beta$ (FWHM $\mathrm{H} \beta$ ) which is our diagnostic low ionization lines observable from the ground out to $\mathrm{z} \approx 0.9$ with optical and (intermittently to $z=3.7$ ) with infrared spectroscopy. It (and/or FWHM MgII $\lambda 2800$ ) is thought to be a measure of assumed virialized motions in the $\mathrm{AD}$ and is thus crucial for estimating black hole masses for large samples of quasars.

- Ratio of the equivalent width of the $4570 \AA$ optical FeII blend and broad $\mathrm{H} \beta\left(R_{\mathrm{FeII}}=\mathrm{W} 4570 \mathrm{FeII}\right.$ blend/W $\mathrm{H} \beta$ ). It is sensitive to the ionization state, the electron density and column density of the BLR gas arising, as far as we can tell, in (at least part of) the gas that produces $\mathrm{H} \beta$ (e.g., $\mathrm{Hu}$ et al. 2008, Marziani et al. 2010).

- Centroid shift at FWHM of the high ionization line CIV $\lambda 1549$ (at half, maximum, c $(1 / 2)$ ). It is a strong diagnostic of winds/outflows (e.g., Marziani et al. 1996: Leighly and Moore 2004).

- Soft X-ray photon index $\left(\Gamma_{\mathrm{S}}\right)$. Most likely an indicator of optically thick Comptonized radiation, and probably a diagnostic of the thermal emission from the AD or of a Compton-thick corona above the disk (Done et al. 2012, Haardt and Maraschi 1991; Jin et al. 2009).

The 4DE1 approach allows the definition of spectral types following source occupation in the optical 4DE1 plane $\mathrm{FWHM}(\mathrm{H} \beta)$ vs $R_{\text {FeII }}$ (Sulentic et al. 2002) and can be extended to include all four dimensions (e.g., Bachev et al. 2004, for CIV 21549). It is possible to identify two populations along the sequence: Population A with $\mathrm{FWHM}(\mathrm{H} \beta) \leq 4000 \mathrm{~km} \mathrm{~s}^{-1}$, and a second Population B of sources with broader lines (see Sulentic et al. 2007, 2011, for the rationale behind two distinct quasar populations).

\section{The phenomenology of the $\mathrm{H} \beta$ emission line profile in the $4 \mathrm{DE} 1$ context}

It is customary to distinguish between broad and narrow components of Balmer lines, with Type 1 AGN showing a non-zero broad component (the case closest to type 2 is type 1.9 , for which the $\mathrm{BC}$ is visible in $\mathrm{H} \alpha$ but not in $\mathrm{H} \beta$ Osterbrock 1981). It is widely believed that the emitting region of these sources suffers heavy obscuration Antonucci and Miller 1985; Antonucci 1993). In some cases - but apparently not in all sources (Pappa et al. 2001; Wolter et al. 2005; Bianchi et al. 2008:(Petrov 2009) - a BC becomes visible in polarised light. In the following we restrict attention to sources that do not show strong evidence of obscuration in their line profiles (i.e., we will exclude type 1.5, 1.8 and 1.9 sources of the old Osterbrock's classification).

Before 4DE1, several workers provided excellent data on $\mathrm{H} \beta$ (e.g., Shuder 1981; Stirpe 1990) but their interpretation was limited by the lack of any contextualization. Without contextualization every quasar is an island under itself without a clear understanding of how it fits into the general population. The FWHM $\mathrm{H} \beta$ distribution is puzzling since its includes profiles over a very wide range of widths, from less than 1000 to more than $20000 \mathrm{~km} \mathrm{~s}^{-1}$. These line width limits can be related to physical processes, although under several assumptions (Laor 2003) that may be justified only after a first organization of empirical properties is accomplished. A first attempt to describe in a systematic way the shape of broad lines in low- $z$ quasars devised a qualitative assignment based on peak shift and shape asymmetry: for example, a redward asymmetric profile with a redshifted peak would be classified as AR,R, a symmetric unshifted as S, etc. (Sulentic 1989). This and more quantitative analyses of the line wing shape stressed the systematic changes occurring in large samples (Boroson and Green 1992, Robinson 1995). Further work of our group involved the analysis of observations collected over a decade and presented in Marziani et al. (2003a). Results from the analysis of those data were later confirmed by a larger sample of 470 bright quasars from SDSS DR5 (Zamfir et al. 2010). The main findings can be summarised as follows:

- There is a trend in $\mathrm{H} \beta$ line profile properties that is correlated with the $4 \mathrm{DE} 1$ sequence Sulentic et al. 2007). Line profile shapes change along the sequence, from a Lorentzian with a blue shifted excess at the line base to a redward asymmetric profile best fit with 2 Gaussian components. The latter profile is typical of the larger FWHM $\gtrsim 4000 \mathrm{~km} \mathrm{~s}^{-1}$. Fig. 1 shows the spectral types at the opposite ends of the sequence. In spectral type A4 (at the high FeII end 

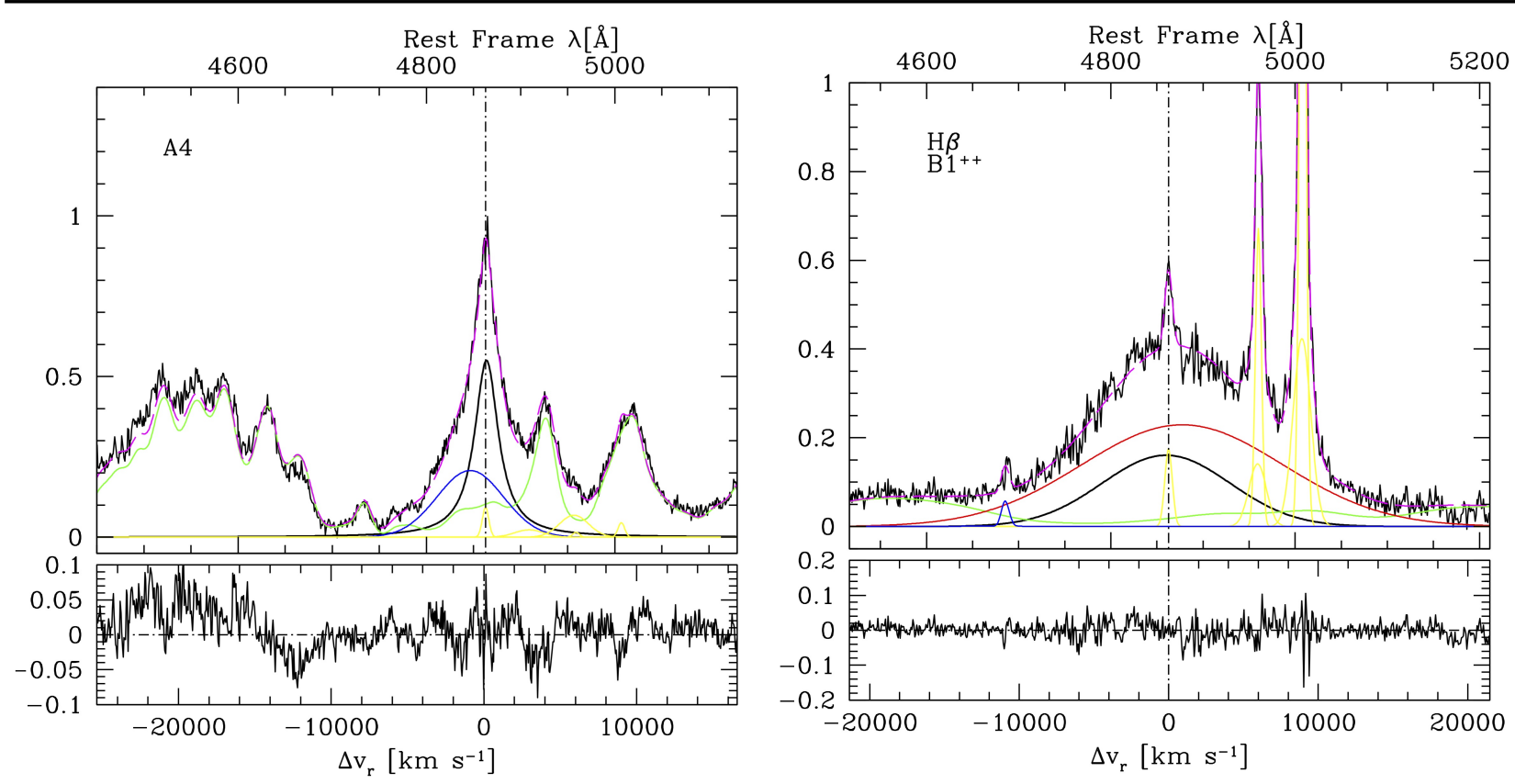

Fig. 1 Median composite $\mathrm{H} \beta$ profiles at the extreme ends of the 4DE1 sequence: spectral type A4 (left), spectral type $(\mathrm{B} 1++)$, from the sample of Marziani et al. (2013a). The full specfit model of the spectrum is traced by the magenta dotted line. Narrow lines are colored yellow, the BC black, the blue shifted component blue, and the VBC red. The Fe II emission (very strong in A4 but almost undetectable in $\mathrm{B} 1++$ ) is coloured pale green. Abscissae are rest frame wavelength in $\AA$ and radial velocity from the peak position of $\mathrm{H} \beta_{\mathrm{NC}}$. The bottom panels show the difference between the spectrum and its model.

of the $4 \mathrm{ED} 1$ sequence) the blue excess of $\mathrm{H} \beta$ is especially evident, as the shallow, redward very broad profile is for $\mathrm{B} 1++$ (at the opposite end of the sequence, with broadest lines and very weak FeII emission).

- Peculiar, double- or multiple peaked profiles are more frequent toward the extreme Pop. B spectral types i.e., B1++ with FWHM $(\mathrm{H} \beta) \geq 12000 \mathrm{~km} \mathrm{~s}^{-1}$.

- Radio loud sources are predominantly Pop. B (Marziani et al. 1996, 2001; Sulentic et al. 2003. Rokaki et al. 2003: Zamfir et al. 2008); radio coredominated (CD) sources show systematically narrower profiles (and distribute in 4DE1 spaced like radio-quiet sources), while lobe-dominated (LD assumed to be Fanaroff-Riley II,FRII) are frequently associated with very broad profiles (almost exclusively Population B).

Peculiar profiles can be empirically grouped in three large categories: (1) double peaked profiles with small peak separation $\left(\lesssim 10^{3} \mathrm{~km} \mathrm{~s}^{-1}\right)$. These sources are apparently rare, since only a handful are known. We stress the apparently since some cases could be masked by the blending of $\mathrm{H} \beta_{\mathrm{BC}}$ and $\mathrm{H} \beta_{\mathrm{NC}}$. Prototypes include Akn 120, IC 4329A, and OX 169 (Marziani et al. 1992); (2) wide separation double peaked profiles $\left(\Delta v \gtrsim 10^{3}\right.$ $\mathrm{km} \mathrm{s}^{-1}$ ), whose prototype are Arp 120B and 3C 390.3
(Chen et al. 1989, Zhang 2011). An example is shown in Fig. 2. (3) Single peaked profiles with a large shift, either to the blue or to the red (two examples, one for each case, are also shown in Fig. 2). A prototypical source, Mkn 668 (Marziani et al. 1993), shows a peak displacement of $+3000 \mathrm{~km} \mathrm{~s}^{-1}$. Strateva et al. (2003) found that class (2) profiles are also relatively rare ( $\lesssim 2 \%$, considering that most of the Strateva et al. 2003 sources are not actually double-peaked sources). The prevalence is higher among RL but significant also among RQ sources.

\subsection{Empirical Interpretation of the $\mathrm{H} \beta$ emission line profile along the $4 \mathrm{DE} 1$ sequence}

Attempts at interpretation of single emission lines are doomed to provide rather ambiguous results. The emitting region is not spatially resolved, and the profiles are usually smooth. They can be described by simple functional forms (logarithmic, or Lorentzian), and these profile can be modelled assuming widely different structural and dynamical conditions. On the converse, comparison of representative HILs to representative LILs ( $\mathrm{H} \beta$ and MgII $\lambda 2800$ ) provides important insights (Marziani et al. 1996). Some $\mathrm{H} \beta$ profiles show a significant excess with respect to a symmetric Lorentzian 
especially in the spectral types associated with the highest $L / L_{\text {Edd }}$ (Marziani et al. 2013b). The blue shifted emission has been interpreted as an outflowing component which has a physical correspondence if there is e.g. a non-rotating radiation driven wind (Elvis 2000).

Further interpretation of the broad profiles involves "stratification" of the emitting region: we associate (likely an over-simplification of a continuous radial trend) the broad component with a lower ionization Broad Line Region (BLR), where line broadening is predominantly virial, and where FeII, CaII are also emitted Martínez-Aldama et al. 2015, and references therein). Consistently, there is no significant shift $\left(\lesssim 200 \mathrm{~km} \mathrm{~s}^{-1}\right.$ ) between $\mathrm{H} \beta$ BC and FeII optical emission (Sulentic et al. 2012). The very broad component is associated with an inner region of higher ionization, the Very Broad Line Region (VBLR) emitting no FeII and showing lower continuum responsivity (Snedden and Gaskell 2007; Goad and Korista 2014). The typical decomposition $\mathrm{BC} / \mathrm{VBC}$ is shown by the mock profile in Fig. 3 as well as by the composite profile in Fig. 1 . LIL profiles often show asymmetries but the amplitude of the asymmetries and shifts is usually much less than their FWHM (Sulentic|1989, Marziani et al.|2003a. Zamfir et al.|2010). The stratification view is consistent with virial motions dominating both the core and the wings of the Pop. B LILs, although the redward asymmetry of Balmer and other LILs is difficult to explain.

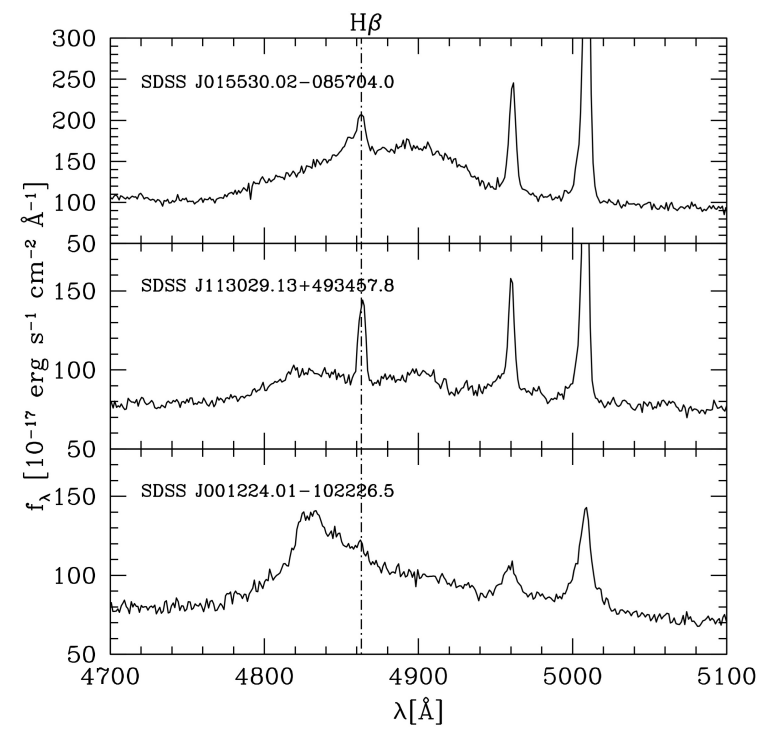

Fig. 2 Three examples of peculiar $\mathrm{H} \beta$ profiles from the sample of Zamfir et al. (2010). Abscissa is rest frame wavelength in $\AA$, ordinate is specific flux in $10^{-17} \mathrm{ergs} \mathrm{s}^{-1} \mathrm{~cm}^{-2}$ $\AA^{-1}$.
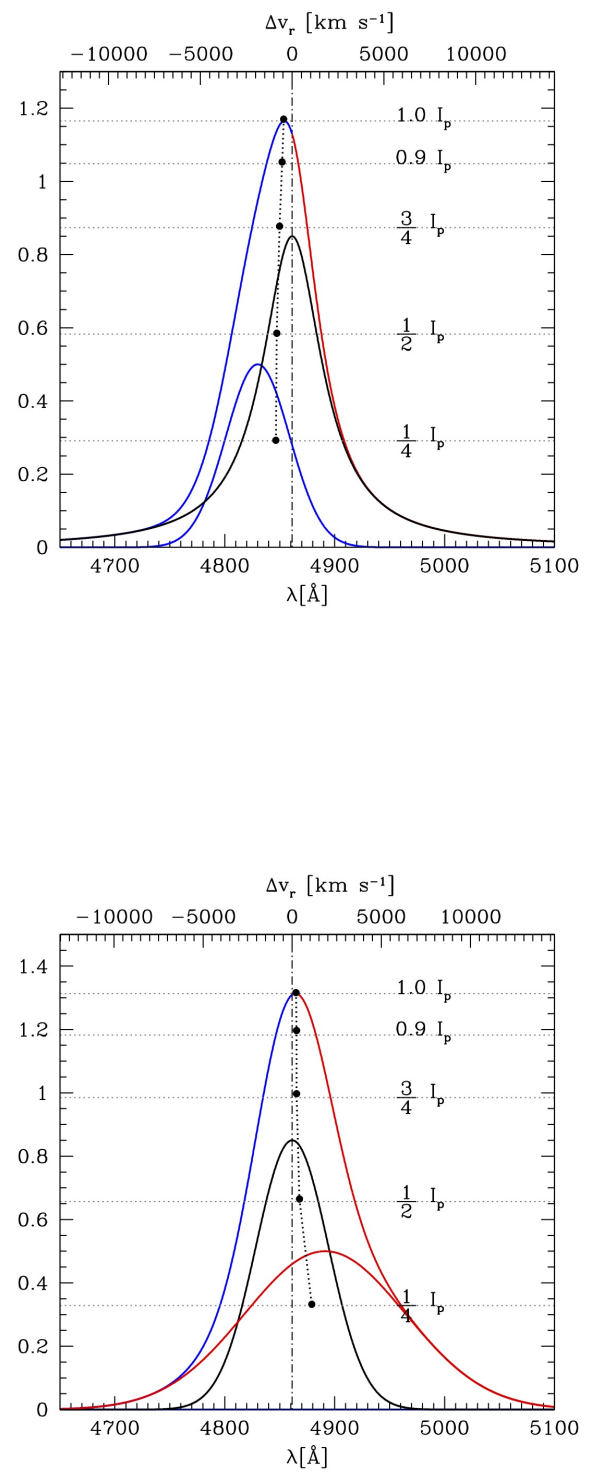

Fig. 3 Mock $\mathrm{H} \beta$ profile illustrating the line decomposition into a BC and a blueshifted component for Pop. A (top), and into a BC, VBC and blue shifted component for Pop. B (bottom). Radial velocities are measured for the peak intensity $I_{\mathrm{p}}$, and the centroids (black spots) at different fractional intensities on the full profile, to obtain a quantitative parameterisation that is independent of the decomposition of the profile. 
3.2 Luminosity and radio-loudness effects on the $\mathrm{H} \beta$ line profile

The main luminosity effect on $\mathrm{H} \beta$ is a systematic increase in the minimum FWHM (Marziani et al. 2009) which has been shown in several papers since 2009 and will not be shown again. At the same time we see that shifts toward the red close to the line base, at $1 / 4$ fractional intensity become more frequent in higher $L$ sources (this is reflected in an increased redward asymmetry). At present we do not see any strong RL effect on $\mathrm{H} \beta$. The median $\mathrm{H} \beta$ line profiles for $\mathrm{RL}$ and RQ Pop. B sources are practically indistinguishable (Marziani et al. 2003b Zamfir et al. 2008). Extreme cases of redward asymmetry are found among radio loud quasars but there is no doubt that Pop. B RQ sources are also capable of impressive redward asymmetries (especially at high luminosity) with $\mathrm{AI} \approx 0.5$. Most of the quasars in our high $L$ sample (Marziani et al. 2009) are RQ quasars (Fig. 4).

As mentioned, RL effects on LILs can be viewed as second order, if they exist at all. In the plane $\mathrm{c}(1 / 4)$ vs FWHM $\mathrm{H} \beta$ the bulk of the radio loud sources occupies the same area of RQ Pop. B, with some CDs trespassing to the Pop. A side, most likely because of an orientation effect, as they are viewed close to the radio axis (Fig. 5). The distribution of redshifts at $1 / 4$ peak intensity shows no difference for Pop. B RQ and $\mathrm{RL}$, if measurements uncertainties are taken into account (lower panel of Fig. 5). There is no correlation with radio-loudness parameters. The panels of Fig. 6 shows that the $\mathrm{H} \beta$ AI does not depend neither on $R_{\mathrm{K}}$ nor on specific radio power.

The behaviour of LILs is definitely different from the one of HILs, where the suppression of CIV $\lambda 1549$ blueshifts occurs mainly in RL sources, making the redward asymmetry associated with the VBC more prominent. In RQ sources, the redward asymmetry is masked by some blue shifted emission. The VBLR is believed by some to be a source of strong CIV $\lambda 1549$ emission (Marziani et al. 2010). The difference between the $\mathrm{H} \beta$ and Civ $\lambda 1549$ profiles can be explained, at least in part, by adding blue shifted CIV $\lambda 1549$ emission in RQ sources.

\section{The physical and dynamical interpretation of line shifts}

\subsection{Atomic physics}

Line broadening and shifts can be produced because of particular physical conditions, for example, for a

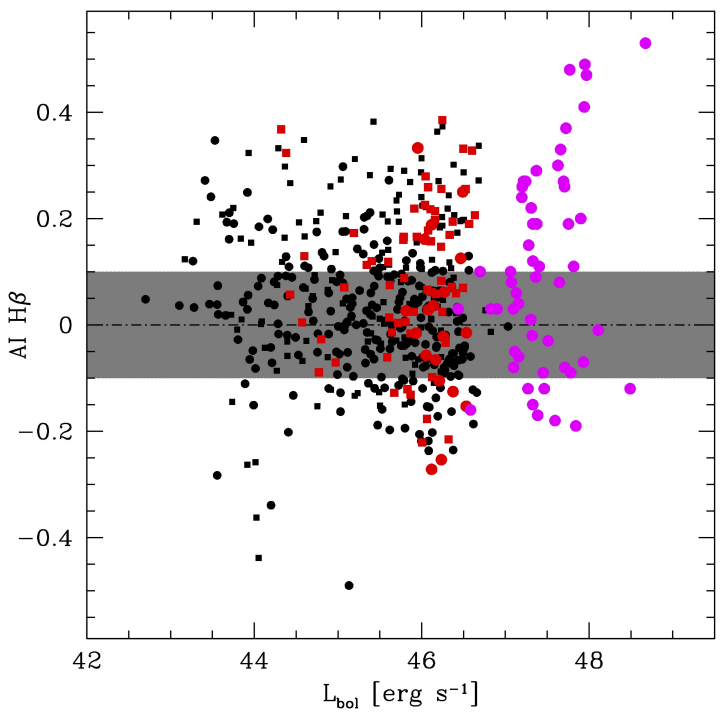

Fig. 4 Luminosity effects on line profiles shapes: the $\mathrm{H} \beta$ AI behaviour as a function of bolometric luminosity in ergs $\mathrm{s}^{-1}$. Black symbols: RQ, red symbols: RL, squares: Pop. B, circles: Pop. A. from the sample of Zamfir et al. (2010). The magenta points at high $L_{\text {bol }}$ represent the sample of Marziani et al. (2009). The shaded band width is set by the uncertainty at a $\pm 2 \sigma$ confidence level in individual AI measurements. Within this band, profiles can be considered symmetric. 
medium at very high pressure such as in white dwarf atmospheres (Halenka et al. 2015, and references therein) by quantum mechanical effects (Wiese and Kelleher 1971). Such shifts are however rather modest even at the highest density. Generally speaking natural and collisional broadening produce Lorentzian profiles (e.g., Peach 1981). We find many Balmer line profiles in Pop. A sources that are Lorentzian-like (to be more precise, they are Voigt due to the convolution with the (Gaussian) instrumental profile). The width is however too large to be produced by collisional effects; Lorentzian profiles are possible also for processes associated with gas motion and geometry.

In principle, thermal broadening could yield a Gaussian line with $\mathrm{FWHM}=\sqrt{8 k T_{e} \ln 2 / m_{e} c^{2}} \lambda_{0}$, where $k$ is the Boltzmann constant $T_{\mathrm{e}}$ the electron temperature, $m_{\mathrm{e}}$ the electron mass, and $\lambda_{0}$ the line rest frame wavelength. For a typical FWHM $\mathrm{H} \beta=4000 \mathrm{~km} \mathrm{~s}^{-1}$, the electron temperature needs to be $\sim 10^{9} \mathrm{~K}$, too high.

Optical depth effects may also produce shifts and absorptions dips in the emission profiles. Narrow dips and not widely-spaced double peaks are sometimes observed in $\mathrm{H} \beta$ profiles (typical cases: IC 4329A, Akn 120, and OX 169 Marziani et al. 1992, Korista 1992 Halpern and Eracleous 2000), but optical depth effects are usually not considered as a possible cause of the absorption, even if the Balmer line optical depth is $\tau \sim 10^{2} \gg 1$. Self-absorption requires that the emitting gas cloud is seen through the line of sight (Smith 1980), which may occur occasionally but not very frequently (a case known for many years involves the $\mathrm{H} \alpha$ profile of NGC 4151, Anderson and Kraft 1969). Accordingly, only a few cases of $\mathrm{H} \beta$ BAL profiles are known (e.g., Aoki et al. 2006, Ji et al. 2012, and references therein). The rarity of these sources is most likely a consequence of the physical conditions needed to produce significant absorption: the maintenance of a large population of HI atoms with electrons excited to level $n=2$ via Ly $\alpha$ resonance scattering, so that the $n=2$ level can become a pseudo-ground level and $\mathrm{H} \beta$ itself "behave" like a resonant line.

\subsection{Scattering processes}

The BLR is believed to be optically thin for electron scattering (Davidson and Netzer 1979). However it is still possible that broad line photons are scattered by hot electron surrounding or embedding the line emitting gas (Shields and McKee 1981), as in the case of an hot inter cloud medium or of an ionised atmosphere above a dense accretion disk. Support for the latter hypothesis has been found by spectropolarimetric analysis of the broad $\mathrm{H} \alpha$ profile: in about ten objects, the PA of
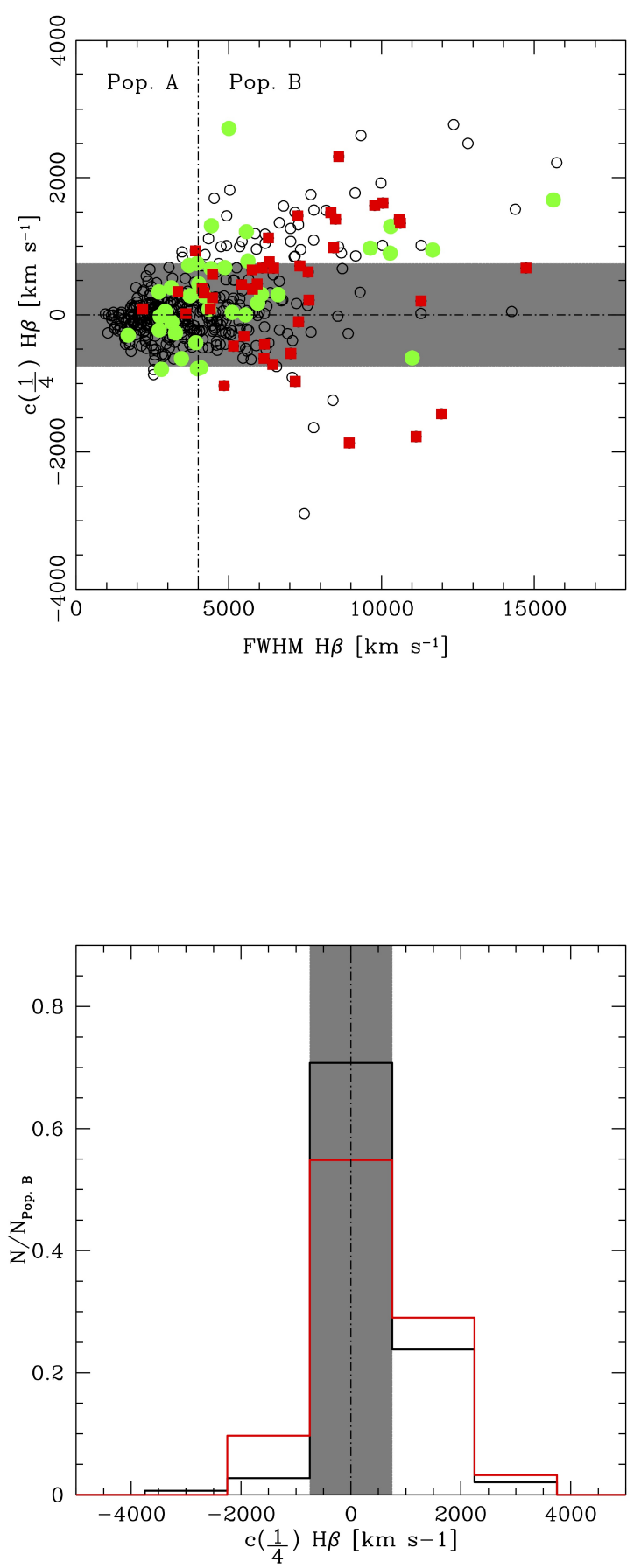

Fig. 5 Top: FWHM H $\beta$ vs c(1/4) parameter in $\mathrm{km} \mathrm{s}^{-1}$ for RQ (open circles), core-dominated (green squares) and FRII (red circles) in the Zamfir et al. (2008) sample. The shaded band width is set by the uncertainty at a $\pm 2 \sigma$ confidence level in individual AI measurements, as in the previous figure. Bottom: Histogram of the $\mathrm{c}(1 / 4)$ distribution for Pop. B sources, with bin width corresponding to the $2 \sigma$ uncertainty associated with $\mathrm{c}(1 / 4)$, for $\mathrm{RQ}$ and $\mathrm{RL}$ (red). The restriction to Pop. B is motivated by the paucity of RL sources in Pop. A. 

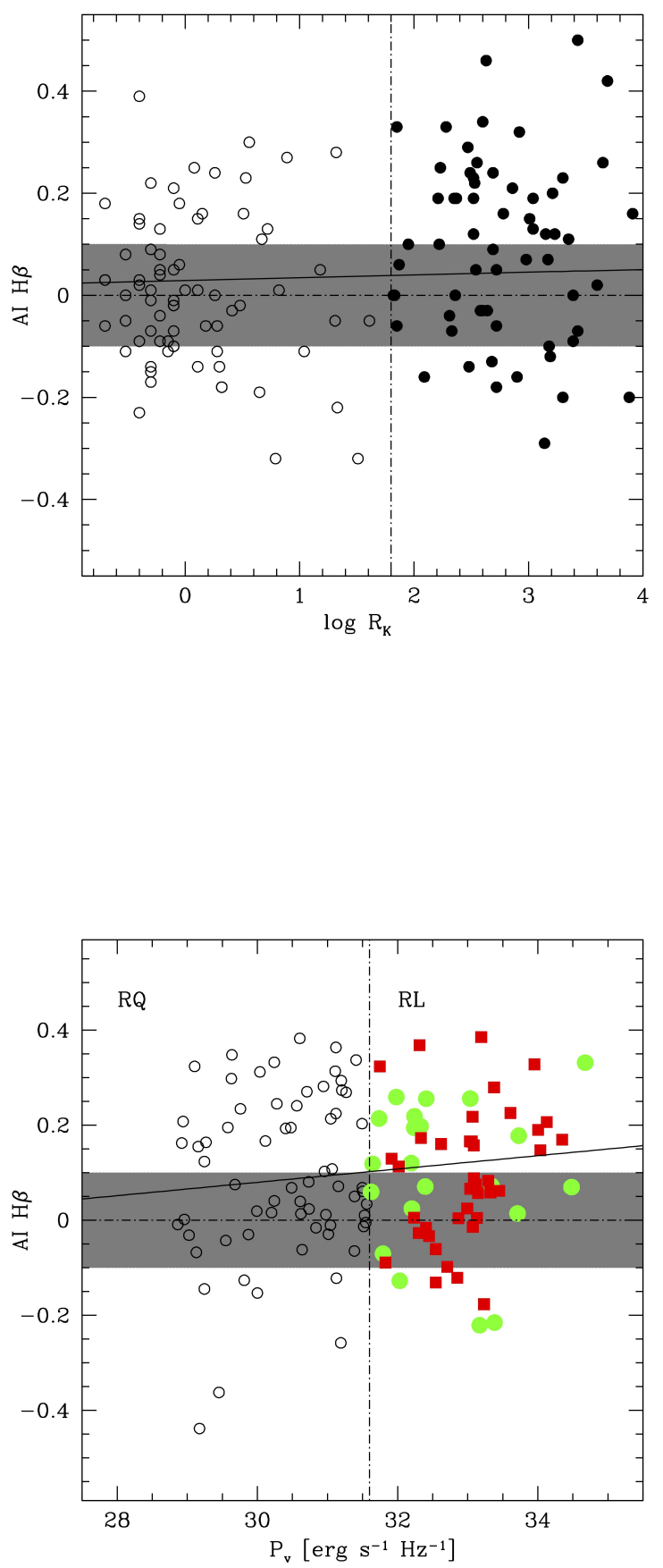

Fig. 6 Radio-loudness effects on $\mathrm{H} \beta$ line profiles. Top: $\mathrm{H} \beta$ AI vs. radio-loudness parameter $R_{\mathrm{K}}$, for radio-detected RQ quasars (open circles) and RL quasars (filled circles) from the sample of Marziani et al. (2003a). The vertical line shows the nominal boundary for RL objects. An unweighted lsq fit yields a trend with a slope not significantly different from 0 (straight red line). Bottom: AI vs. radio power at $20 \mathrm{~cm}$ for Pop. B sources in the sample of Zamfir et al. (2008). Meaning of symbols is the same as in Fig. 5 The slope of the straight line is $0.14 \pm 0.10$, again not significantly different from 0 . the polarisation vector rotates across the profile, reaching opposite PA in the red and blue wings (Smith et al. 2005), as expected if light is scattered from a rotating disk. Since the emitting region radius is now believed to be much smaller than in the past (the inner radius might be less than $100 \mathrm{R}_{\mathrm{g}}$ ), it is possible that the innermost broad line emission, already associated with gas in rapid motion, is further broadened by a screen of hot electrons (Laor 2006). It is intriguing that Smith et al. (2005) found lower polarisation in the line core. This is consistent with a two component model (i.e., $\mathrm{BC}+$ $\mathrm{VBC}$ ) where the core is emitted further out than the line wings, possibly outside of the screen of scattering material.

Other scattering processes might be operating as well. Rayleigh scattering could be relevant if there is neutral material of high column density, as might be present in an accretion disk or a molecular torus. It could be more important than electron scattering in the UV (Gaskell and Goosmann 2013). Rayleigh scattering could contribute the broadening of the extended line wings in $\operatorname{Ly} \alpha$ if $\operatorname{Ly} \alpha$ is emitted above the disk and scattered towards the observer by the dense accretion disk (Lee 2005).

Fast (relativistic) electrons moving in a dense medium could be give rise to Cerenkov line-like radiation (You et al. 1986, Cheng et al. 1990). Densities above $n_{\mathrm{H}} \gtrsim 10^{13} \mathrm{~cm}^{-3}$ are required for a significant effect. This may be the case for an accretion disk as well as for dense gas clumps emitting the LILs in extreme Population A sources (Negrete et al. 2012). Shifts on the order of $\sim 1000 \mathrm{~km} \mathrm{~s}^{-1}$ should appear as a net redshift in the Balmer and Lyman lines (Liu et al. 2014).

\subsection{Gravitational redshift}

An early analysis of gravitational redshift in the context of broad line emission was given by Netzer (1977). A detailed discussion of gravitational redshift effects on the Balmer line profiles is provided by Bon et al. (submitted for this volume). Here we just recall that gravitation can induce a shift $\Delta z \approx \frac{G M_{\mathrm{BH}}}{R c^{2}}=\frac{1}{2} \frac{R_{\mathrm{S}}}{R}$ where $M_{\mathrm{BH}}$ is the black hole mass, and $R_{\mathrm{S}}$ the Schwarzschild radius $\left(2 G M_{\mathrm{BH}} / c^{2}\right)$. This relation is valid with an approximation of $4 \%$ down to a 20 Schwarzschild radii. The total shift of line profiles would include the effect of transverse Doppler redshift, so that the expected shift is $\Delta z \approx \frac{3}{4} \frac{R_{\mathrm{S}}}{R}$. Two considerations are in order:

- the most massive BHs may be associated with the strongest red-ward asymmetries in the $\mathrm{H} \beta$ line (Marziani et al. 2009);

- ray tracing in Kerr metrics produce red wings that reproduce fairly well the observations of observed profiles in several high-luminosity sources. 
Is this enough to justify the claim that grav $z$ produces the redward asymmetry in Pop. B sources? The answer is "No". The problem is that the models assume disk emission with steep emissivity laws that give a significant weight to the inner disk regions. Since the radii needed to explain the centroid shifts at $1 / 4$ or the VBC displacements are $\sim 100 R_{S}$, there is not likely to be enough cold gas to explain the most luminous sources. A more detailed model that includes both kinematics and emitting gas physical conditions (assuming, for instance, that the gas in confined to a dense disk) is needed before a conclusion can be reached (Bon et al.).

\subsection{Doppler effect due to an ordered velocity field}

There are several lines of evidence supporting virial motion as an important source of line broadening:

- velocity resolved reverberation mapping studies (Koratkar and Gaskell 1991; Grier et al. 2013) exclude outflows as the broadening source at least in the LILs of Population B sources.

- an anti-correlation between size (derived from reverberation mapping) and line width found for several lines in the same sources (Peterson and Wandel 1999): a central massive object gravitationally dominates cloud motions, with the lines emitted by ionic species of higher ionization potential due to gas moving faster, and emitted closer to the central massive object.

- a fairly symmetric profile is by far the most frequent; apart from the peculiar cases, shifts are usually less than 0.1 of the line width at the same fractional height (Sulentic 1989).

- $\mathrm{H} \beta$ and $\operatorname{MgII} \lambda 2800$ show a trend in line width consistent with the domination of virial motions. $\operatorname{MgII} \lambda 2800$ is preferentially emitted at slightly larger distance than $\mathrm{H} \beta$ from the continuum source (Marziani et al. 2013a);

- there is a gradient in ionization level (approximated in the decomposition BLR/VBLR) that is consistent with a virial velocity field.

Extreme population A sources (spectral type A3 and A4) often show a blue-shifted excess indicative of radial motion and most likely associated with an outflow. This said, the geometry of the gas is not yet clear. The fundamental issue of whether the gas is associated with a disk or with a system of clouds is still not settled. Clouds models are still formulated at the time of writing perhaps by authors unaware of the confinement problems that cloud models face (Shadmehri|2015), of which the most serious remains the expected decay time due to the viscous attrition with some putative "inter cloud medium." Disk + wind models provide some consistency with ideas about Pop. B sources (Flohic et al. 2012), but they still fail to reproduce a typical Pop. $\mathrm{B} \mathrm{H} \beta$ profile. At the same time, a Lorentzian profile might be reasonably associated with an extended disk, but other models can also produce Lorentzian or almost Lorentzian shapes, most notably a system of clouds under the combined influence of gravity and radiation pressure forces (Mathews 1993; Netzer and Marziani 2010).

4.5 Peculiar profiles: Binary BLR, disks and/or outflows?

Binary BH The search for binary black holes is still in its infancy. Apart from a few very special cases such as OJ 287 (Sillanpaa et al. 1988), evidence in favour of sub-parsec binaries that could significantly affect the broad line profiles is still sparse, and has been hard to find (Bon et al. 2012). Some of the most classical double peaked sources (Arp 102B, Halpern and Filippenko 1988) show remarkable stability of the peak positions over decades, ruling out a binary system with reasonable masses (Gezari et al.|2007). Similar considerations were made for OX 169 (Halpern and Eracleous 2000). Sub-pc BBH should be numerous at high $z$ but rarer in the local Universe (Volonteri et al. 2009). It is still unclear whether we should look for a sizeable minority population or for extremely rare event, as coalescence times depend on the evolution of the binary orbit eccentricity, and circularised orbit are "stiff" since they do not yield to orbital losses due to gravitational radiation. Profiles with large shifts are perhaps more promising (Boroson and Lauer 2009), and extensive searches for eventual monitoring are being carried out (e.g., Ju et al. 2013).

The accretion disk explanation that fits Arp 102B so well fails for other double peaked sources for which a more suitable model may be offered by elliptical disks (Eracleous et al. 1995). Accretion disk emission has been postulated for the broad line wings (Bon et al. 2009). Modelling of a disk may require abandoning the weak field treatment in order to model the red asymmetry of Balmer line profiles in Pop. B (Bon et al. submitted).

Single peaked profiles with large shift can be explained by radially moving gas. However, inferences on the direction of radial motions are affected by isotropy of line emission: if the line is anisotropically emitted as expected for the Balmer lines in the physical conditions of the BLR (Ferland et al. 1992), then a redshift corresponds to inflow (accretion?), and a blueshift to outflow (winds?), although it is still possible that we see emission mainly from the shielded face of clouds (Ferland et al. 2009). 
5 How can LIL shifts and other profile properties help build a physical scenario

Line profiles of Balmer lines are orientation dependent. This result emerges on a statistical basis by comparing the average line widths of lobe-dominated and core-dominated radio-loud sources (Wills and Browne 1986, McLure and Dunlop 2002; Sulentic et al. 2003). Conservation of angular momentum accounts for the the spherical symmetry break that leads to the formation of a Keplerian (or quasi-Keplerian) accretion disk around the black hole. Not surprisingly, a flattened configuration was already "in place" in the minds of astronomers from heuristic considerations during the 1970s (e.g., Osterbrock 1978). However, the viewing angle is a parameter that is generally unknown for RQ sources and can be estimated only for superluminal radio loud sources on an individual basis (Rokaki et al. 2003 Sulentic et al. 2003). Approaches using polarimetry (Afanasiev and Popović 2015) and gravitational redshift (if found appropriate) can in principle lead to an estimate of the viewing angle (Gavrilović et al. 2007 Kollatschny 2003). A flattened or a "bowl"-shaped geometry (Kollatschny and Zetzl 2011; Goad et al. 2012) is suggested by the dependence of line width on viewing angle. Goad et al. (2012) suggested that line emission may occur in the outer region of the accretion disk, where it should merge with the surrounding molecular torus, and were able to obtain Lorentzian profiles for the integrated emission from that region. This model is intriguing and may well be applicable to extreme Pop. A sources.

Heuristic considerations and black hole and Eddington ratio estimates suggest that the main driver of the quasar main sequence is Eddington ratio (e.g., Marziani et al. 2001, 2003b Kuraszkiewicz et al. 2009). Population $\mathrm{A}$ and $\mathrm{B}$ are distinct in terms of Eddington ratio: the boundary value (for $\mathrm{BH}$ mass of $10^{8} \mathrm{M}_{\odot}$ ) is estimated to be $L / L_{\mathrm{Edd}} \approx 0.2 \pm 0.1$ (Marziani et al. 2003b). It is interesting to note that this is the limit at which the transition from a geometrically thin to a geometrically thick disk is expected (Abramowicz et al. 1988, Frank et al. 2002 and references therein). So, any model of the BLR should be based on this constraint, with models valid for Pop. A including a geometrically thick disk, its anisotropy and shadowing effects. A first attempt to include a geometrically thick disk in a BLR model has been carried out by Wang et al. (2014), but the predictions until now contradict the observations.

\section{Key Open issues and conclusions}

It is perhaps disappointing that after 40 years of effort the structure and dynamics of the regions emitting the
LILs are poorly understood. However, on the bright side, some key points have been clarified:

- we now have a contextualization which allows us to relate, and discriminate between source classes;

- a trend in Balmer line shapes is systematic along the 4DE1 sequence;

- Lorentzian (Pop. A) or double Gaussian (Pop. B) are by far the most frequent $\mathrm{H} \beta$ and $\operatorname{MgII} \lambda 2800$ profiles;

- only a minority of sources show very broad profiles that are immediately consistent with accretion disk emission;

- the wide range of HIL FWHM suggests that black hole mass estimates using this measure as a virial estimator must be treated with caution. No model can explain this range invoking only line of sight orientation of a flattened disk. The rules for mass estimation are likely different for Pop. A and B sources.

The profile differences may be associated with an accretion state transition.

Degeneracy of the regular profile with respect to competing models is still a major problem. Knowing however that $L / L_{\mathrm{Edd}}$ is a major driver in HIL profile diversity along the 4DE1 sequence will help building more focused models.

AdO and JS acknowledge the support by the Junta de Andalucía through project TIC114,and the Spanish Ministry of Economy and Competitiveness (MINECO) through project AYA2013-42227-P. 


\section{References}

Abramowicz, M.A., Czerny, B., Lasota, J.P., Szuszkiewicz, E.: Astrophys. J. 332, 646 (1988). doi:10.1086/166683

Afanasiev, V.L., Popović, L.Č.: Astrophys. J. Lett. 800, 35 (2015). 1501.07730. doi:10.1088/2041-8205/800/2/L35

Anderson, K.S., Kraft, R.P.: Astrophys. J. 158, 859 (1969). doi:10.1086/150246

Antonucci, R.: Annu. Rev. Astron. Astrophys. 31, 473 (1993). doi:10.1146/annurev.aa.31.090193.002353

Antonucci, R.R.J., Miller, J.S.: Astrophys. J. 297, 621 (1985). doi:10.1086/163559

Aoki, K., Iwata, I., Ohta, K., Ando, M., Akiyama, M., Tamura, N.: Astrophys. J. 651, 84 (2006). arXiv:astroph/0607036. doi:10.1086/507438

Bachev, R., Marziani, P., Sulentic, J.W., Zamanov, R., Calvani, M., Dultzin-Hacyan, D.: ApJ 617, 171 (2004). arXiv:astro-ph/0408334. doi:10.1086/425210

Bianchi, S., Corral, A., Panessa, F., Barcons, X., Matt, G., Bassani, L., Carrera, F.J., Jiménez-Bailón, E.: Mon. Not. R. Astron. Soc. 385, 195 (2008). 0710.4226. doi:10.1111/j.1365-2966.2007.12625.x

Bon, E., Popović, L.Č., Gavrilović, N., Mura, G.L., Mediavilla, E.: Mon. Not. R. Astron. Soc. 400, 924 (2009). 0908.2939. doi:10.1111/j.1365-2966.2009.15511.x

Bon, E., Jovanović, P., Marziani, P., Shapovalova, A.I., Bon, N., Borka Jovanović, V., Borka, D., Sulentic, J., Popović, L.Č.: Astrophys. J. 759, 118 (2012). 1209.4524. doi:10.1088/0004-637X/759/2/118

Boroson, T.A., Green, R.F.: ApJS 80, 109 (1992). doi:10.10$86 / 191661$

Boroson, T.A., Lauer, T.R.: Nature 458, 53 (2009). 0901.3779. doi:10.1038/nature07779

Chen, K., Halpern, J.P., Filippenko, A.V.: Astrophys. J. 339, 742 (1989). doi:10.1086/167332

Cheng, F.H., You, J.H., Yan, M.: Astrophys. J. 358, 18 (1990). doi:10.1086/168958

Collin-Souffrin, S., Dyson, J.E., McDowell, J.C., Perry, J.J.: MNRAS 232, 539 (1988)

Corbin, M.R., Boroson, T.A.: Astrophys. J. Suppl. Ser. 107, 69 (1996). doi:10.1086/192355

Davidson, K., Netzer, H.: Reviews of Modern Physics 51, 715 (1979). doi:10.1103/RevModPhys.51.715

Done, C., Davis, S.W., Jin, C., Blaes, O., Ward, M.: Mon. Not. R. Astron. Soc. 420, 1848 (2012). 1107.5429. doi:10.1111/j.1365-2966.2011.19779.x

D'Onofrio, M., Marziani, P., Sulentic, J.W. (eds.): Fifty Years of Quasars From Early Observations and Ideas to Future Research. Astrophysics and Space Science Library, vol. 386. Springer Verlag, Berlin-Heidelberg, ??? (2012)

Elvis, M.: Astrophys. J. 545, 63 (2000). arXiv:astroph/0008064. doi:10.1086/317778

Eracleous, M., Livio, M., Halpern, J.P., Storchi-Bergmann, T.: Astrophys. J. 438, 610 (1995). doi:10.1086/175104

Ferland, G.J., Peterson, B.M., Horne, K., Welsh, W.F., Nahar, S.N.: Astrophys. J. 387, 95 (1992). doi:10.1086/171063

Ferland, G.J., Hu, C., Wang, J., Baldwin, J.A., Porter, R.L., van Hoof, P.A.M., Williams, R.J.R.: Astrophys. J. Lett. 707, 82 (2009). 0911.1173. doi:10.1088/0004$637 \mathrm{X} / 707 / 1 /$ L82
Flohic, H.M.L.G., Eracleous, M., Bogdanović, T.: ApJ 753, 133 (2012). doi:10.1088/0004-637X/753/2/133

Frank, J., King, A., Raine, D.J.: Accretion Power in Astrophysics: Third Edition, Iii edition edn. Cambridge University Press, ??? (2002)

Gaskell, C.M.: ApJ 263, 79 (1982). doi:10.1086/160481

Gaskell, C.M., Goosmann, R.W.: Astrophys. J. 769, 30 (2013). 0805.4258. doi:10.1088/0004-637X/769/1/30

Gavrilović, N., Popović, L.Č., Kollatschny, W.: In: Karas, V., Matt, G. (eds.) IAU Symposium. IAU Symposium, vol. 238, p. 369 (2007). doi:10.1017/S1743921307005492

Gezari, S., Halpern, J.P., Eracleous, M.: Astrophys. J. Suppl. Ser. 169, 167 (2007). arXiv:astro-ph/0702594. doi: $10.1086 / 511032$

Goad, M.R., Korista, K.T.: Mon. Not. R. Astron. Soc. 444, 43 (2014). 1407.5004. doi:10.1093/mnras/stu1456

Goad, M.R., Korista, K.T., Ruff, A.J.: Mon. Not. R. Astron. Soc. 426, 3086 (2012). 1207.6339. doi:10.1111/j.1365-2966.2012.21808.x

Greenstein, J.L., Schmidt, M.: Astrophys. J. 140, 1 (1964). doi:10.1086/147889

Grier, C.J., Peterson, B.M., Horne, K., Bentz, M.C., Pogge, R.W., Denney, K.D., De Rosa, G., Martini, P., Kochanek, C.S., Zu, Y., Shappee, B., Siverd, R., Beatty, T.G., Sergeev, S.G., Kaspi, S., Araya Salvo, C., Bird, J.C., Bord, D.J., Borman, G.A., Che, X., Chen, C., Cohen, S.A., Dietrich, M., Doroshenko, V.T., Efimov, Y.S., Free, N., Ginsburg, I., Henderson, C.B., King, A.L., Mogren, K., Molina, M., Mosquera, A.M., Nazarov, S.V., Okhmat, D.N., Pejcha, O., Rafter, S., Shields, J.C., Skowron, J., Szczygiel, D.M., Valluri, M., van Saders, J.L.: Astrophys. J. 764, 47 (2013). 1210.2397. doi:10.1088/0004$637 \mathrm{X} / 764 / 1 / 47$

Haardt, F., Maraschi, L.: Astrophys. J. Lett. 380, 51 (1991). doi:10.1086/186171

Halenka, J., Olchawa, W., Madej, J., Grabowski, B.: Astrophys. J. 808, 131 (2015). 1506.04064. doi:10.1088/0004$637 \mathrm{X} / 808 / 2 / 131$

Halpern, J.P., Eracleous, M.: Astrophys. J. 531, 647 (2000). astro-ph/9910118. doi:10.1086/308516

Halpern, J.P., Filippenko, A.V.: Nature 331, 46 (1988). doi: $10.1038 / 331046 \mathrm{a} 0$

Hu, C., Wang, J.-M., Ho, L.C., Chen, Y.-M., Bian, W.-H., Xue, S.-J.: ApJL 683, 115 (2008). 0807.2060. doi: $10.1086 / 591848$

Ji, T., Wang, T.-G., Zhou, H.-Y., Wang, H.-Y.: Research in Astronomy and Astrophysics 12, 369 (2012). 1201.1054. doi:10.1088/1674-4527/12/4/002

Jin, C., Done, C., Ward, M., Gierliński, M., Mullaney, J.: Mon. Not. R. Astron. Soc. 398, 16 (2009). 0903.4698. doi:10.1111/j.1745-3933.2009.00697.x

Ju, W., Greene, J.E., Rafikov, R.R., Bickerton, S.J., Badenes, C.: Astrophys. J. 777, 44 (2013). 1306.4987. doi:10.1088/0004-637X/777/1/44

Kollatschny, W.: Astron. Astrophys. 407, 461 (2003). astroph/0306389. doi:10.1051/0004-6361:20030928

Kollatschny, W., Zetzl, M.: Nature 470, 366 (2011). doi:10.1038/nature09761

Koratkar, A.P., Gaskell, C.M.: Astrophys. J. Suppl. Ser. 75, 719 (1991). doi:10.1086/191547 
Korista, K.T.: Astrophys. J. Suppl. Ser. 79, 285 (1992). doi:10.1086/191654

Kuraszkiewicz, J., Wilkes, B.J., Schmidt, G., Smith, P.S., Cutri, R., Czerny, B.: Astrophys. J. 692, 1180 (2009). doi:10.1088/0004-637X/692/2/1180

Laor, A.: Astrophys. J. 590, 86 (2003). astro-ph/0302541. doi: $10.1086 / 375008$

Laor, A.: Astrophys. J. 643, 112 (2006). astro-ph/0601688. doi:10.1086/502798

Lee, H.-W.: In: Adamson, A., Aspin, C., Davis, C., Fujiyoshi, T. (eds.) Astronomical Polarimetry: Current Status and Future Directions. Astronomical Society of the Pacific Conference Series, vol. 343, p. 441 (2005)

Leighly, K.M., Moore, J.R.: Astrophys. J. 611, 107 (2004). arXiv:astro-ph/0402453. doi:10.1086/422088

Liu, D.B., Chen, W.P., You, J.H., Chen, L.: Astrophys. J. 780, 89 (2014). doi:10.1088/0004-637X/780/1/89

Loli Martínez-Aldama, M. L., Dultzin, D., Marziani, P., Sulentic, J.W., Bressan, A., Chen, Y., Stirpe, G.M.: Astrophys. J. Suppl. Ser. 217, 3 (2015). 1501.04718. doi:10.1088/0067-0049/217/1/3

Marziani, P., Calvani, M., Sulentic, J.W.: ApJ 393, 658 (1992). doi:10.1086/171533

Marziani, P., Dultzin-Hacyan, D., Sulentic, J.W.: In: Kreitler, P.V. (ed.) Accretion onto Supermassive Black Holes in Quasars: Learning from Optical/UV Observations vol. New Developments in Black Hole Research, p. 123. Nova Press, New York, ??? (2006)

Marziani, P., Sulentic, J.W., Calvani, M., Perez, E., Moles, M., Penston, M.V.: ApJ 410, 56 (1993). arXiv:astroph/9301001. doi:10.1086/172724

Marziani, P., Sulentic, J.W., Dultzin-Hacyan, D., Calvani, M., Moles, M.: ApJS 104, 37 (1996). doi:10.1086/192291

Marziani, P., Sulentic, J.W., Zwitter, T., Dultzin-Hacyan, D., Calvani, M.: ApJ 558, 553 (2001). arXiv:astroph/0105343. doi:10.1086/322286

Marziani, P., Sulentic, J.W., Zamanov, R., Calvani, M., Dultzin-Hacyan, D., Bachev, R., Zwitter, T.: ApJS 145, 199 (2003a). doi:10.1086/346025

Marziani, P., Zamanov, R.K., Sulentic, J.W., Calvani, M.: MNRAS 345, 1133 (2003b). arXiv:astro-ph/0307367. doi:10.1046/j.1365-2966.2003.07033.x

Marziani, P., Sulentic, J.W., Stirpe, G.M., Zamfir, S., Calvani, M.: A\&Ap 495, 83 (2009). 0812.0251. doi:10.1051/0004-6361:200810764

Marziani, P., Sulentic, J.W., Negrete, C.A., Dultzin, D., Zamfir, S., Bachev, R.: Mon. Not. R. Astron. Soc. 409, 1033 (2010). 1007.3187. doi:10.1111/j.13652966.2010.17357.x

Marziani, P., Sulentic, J.W., Plauchu-Frayn, I., del Olmo, A.: AAp (2013a). 1305.1096

Marziani, P., Sulentic, J.W., Plauchu-Frayn, I., del Olmo, A.: ApJ 764(150) (2013b). 1301.0520

Mathews, W.G.: ApJL 412, 17 (1993). doi:10.1086/186929

McLure, R.J., Dunlop, J.S.: Mon. Not. R. Astron. Soc. 331, 795 (2002). astro-ph/0108417. doi:10.1046/j.13658711.2002.05236.x

Negrete, A., Dultzin, D., Marziani, P., Sulentic, J.: ApJ 757, 62 (2012). 1107.3188

Netzer, H.: Mon. Not. R. Astron. Soc. 181, 89 (1977)
Netzer, H., Marziani, P.: Astrophys. J. 724, 318 (2010). 1006.3553. doi:10.1088/0004-637X/724/1/318

Osterbrock, D.E.: Phys. Scr. 17, 285 (1978). doi:10.1088/0031-8949/17/3/024

Osterbrock, D.E.: Publ. Astron. Soc. Pac. 91, 608 (1979)

Osterbrock, D.E.: Astrophys. J. 249, 462 (1981). doi:10.$1086 / 159306$

Pappa, A., Georgantopoulos, I., Stewart, G.C., Zezas, A.L.: Mon. Not. R. Astron. Soc. 326, 995 (2001). astroph/0104061. doi:10.1046/j.1365-8711.2001.04609.x

Peach, G.: Advances in Physics 30, 367 (1981). doi:10.1080/00018738100101467

Peterson, B.M., Wandel, A.: Astrophys. J. Lett. 521, 95 (1999). arXiv:astro-ph/9905382. doi:10.1086/312190

Petrov, G.P.: Bulgarian Astronomical Journal 11, 79 (2009)

Richards, G.T., Kruczek, N.E., Gallagher, S.C., Hall, P.B., Hewett, P.C., Leighly, K.M., Deo, R.P., Kratzer, R.M., Shen, Y.: Astron. J. 141, 167 (2011). 1011.2282. doi:10.1088/0004-6256/141/5/167

Robinson, A.: Mon. Not. R. Astron. Soc. 272, 647 (1995)

Rokaki, E., Lawrence, A., Economou, F., Mastichiadis, A.: Mon. Not. R. Astron. Soc. 340, 1298 (2003). arXiv:astroph/0301405. doi:10.1046/j.1365-8711.2003.06414.x

Shadmehri, M.: Mon. Not. R. Astron. Soc. 451, 3671 (2015). 1506.00247. doi:10.1093/mnras/stv1212

Shields, G.A., McKee, C.F.: Astrophys. J. Lett. 246, 57 (1981). doi:10.1086/183552

Shuder, J.M.: Astrophys. J. 244, 12 (1981). doi:10.1086/158678

Sillanpaa, A., Haarala, S., Valtonen, M.J., Sundelius, B., Byrd, G.G.: Astrophys. J. 325, 628 (1988). doi:10.1086/166033

Smith, H.E.: Astrophys. J. Lett. 241, 137 (1980). doi:10.$1086 / 183377$

Smith, J.E., Robinson, A., Young, S., Axon, D.J., Corbett, E.A.: Mon. Not. R. Astron. Soc. 359, 846 (2005). astroph/0501640. doi:10.1111/j.1365-2966.2005.08895.x

Snedden, S.A., Gaskell, C.M.: ApJ 669, 126 (2007). doi: $10.1086 / 521290$

Stirpe, G.M.: A\&ApS 85, 1049 (1990)

Strateva, I.V., Strauss, M.A., Hao, L., Schlegel, D.J., Hall, P.B., Gunn, J.E., Li, L., Ivezić, Ž., Richards, G.T., Zakamska, N.L., Voges, W., Anderson, S.F., Lupton, R.H., Schneider, D.P., Brinkmann, J., Nichol, R.C.: AJ 126, 1720 (2003). arXiv:astro-ph/0307357. doi:10.1086/378367

Sulentic, J., Marziani, P., Zamfir, S.: Baltic Astronomy 20, 427 (2011)

Sulentic, J.W.: Astrophys. J. 343, 54 (1989). doi:10.1086/167684

Sulentic, J.W., Marziani, P., Dultzin-Hacyan, D.: ARA\&A 38, 521 (2000). doi:10.1146/annurev.astro.38.1.521

Sulentic, J.W., Marziani, P., Dultzin-Hacyan, D., Calvani, M., Moles, M.: ApJL 445, 85 (1995). doi:10.1086/187896

Sulentic, J.W., Marziani, P., Zamanov, R., Bachev, R., Calvani, M., Dultzin-Hacyan, D.: ApJL 566, 71 (2002). arXiv:astro-ph/0201362. doi:10.1086/339594

Sulentic, J.W., Zamfir, S., Marziani, P., Bachev, R., Calvani, M., Dultzin-Hacyan, D.: ApJL 597, 17 (2003). arXiv:astro-ph/0309469. doi:10.1086/379754 
Sulentic, J.W., Bachev, R., Marziani, P., Negrete, C.A., Dultzin, D.: ApJ 666, 757 (2007). 0705.1895. doi:10.10$86 / 519916$

Sulentic, J.W., Zamfir, S., Marziani, P., Dultzin, D.: In: Revista Mexicana de Astronomia y Astrofisica Conference Series. Revista Mexicana de Astronomia y Astrofisica Conference Series, vol. 32, p. 51 (2008)

Sulentic, J.W., Marziani, P., Zamfir, S., Meadows, Z.A.: Astrophys. J. Lett. 752, 7 (2012). 1203.5992. doi:10.1088/2041-8205/752/1/L7

Tytler, D., Fan, X.-M.: ApJS 79, 1 (1992). doi:10.1086/191642

Volonteri, M., Miller, J.M., Dotti, M.: Astrophys. J. Lett. 703, 86 (2009). 0903.3947. doi:10.1088/0004-637X/703/1/L86

Wang, J.-M., Qiu, J., Du, P., Ho, L.C.: Astrophys. J. 797, 65 (2014). 1410.5285. doi:10.1088/0004-637X/797/1/65

Wiese, W.L., Kelleher, D.E.: Astrophys. J. Lett. 166, 59 (1971). doi:10.1086/180739

Wills, B.J., Browne, I.W.A.: Astrophys. J. 302, 56 (1986). doi:10.1086/163973

Wolter, A., Gioia, I.M., Henry, J.P., Mullis, C.R.: Astron. Astrophys. 444, 165 (2005). astro-ph/0510045. doi:10.1051/0004-6361:20053441

You, J.-H., Cheng, F.-H., Cheng, F.-Z., Kiang, T.: Phys. Rev. A 34, 3015 (1986). doi:10.1103/PhysRevA.34.3015

Zamfir, S., Sulentic, J.W., Marziani, P.: MNRAS 387, 856 (2008). 0804.0788. doi:10.1111/j.1365-2966.2008.13290.x

Zamfir, S., Sulentic, J.W., Marziani, P., Dultzin, D.: Mon. Not. R. Astron. Soc. 403, 1759 (2010). 0912.4306. doi:10.1111/j.1365-2966.2009.16236.x

Zhang, X.-G.: Mon. Not. R. Astron. Soc. 416, 2857 (2011). 1107.0455. doi:10.1111/j.1365-2966.2011.19234.x

This manuscript was prepared with the AAS IATEX macros v5.2. 